\title{
Idiopathic postpneumonectomy pulmonary edema: Hyperinflation of the remaining lung is a potential etiologic factor, but the condition can be averted by balanced pleural drainage
}

John M. Alvarez, MBBS, FRACS, ${ }^{a}$ Jeremy Tan, MBBS, ${ }^{a}$ Nand Kejriwal, MBBS, ${ }^{a}$ Karim Ghanim, MBBS, ${ }^{\text {a }}$

Mark A. J. Newman, FRACS, MBBS, ${ }^{a}$ Amanda Segal, FRACP, ${ }^{b}$ Greg Sterret, FRACP, ${ }^{b}$ and Max K. Bulsara, PhD ${ }^{c}$

From the Departments of Cardiothoracic Surgery $^{\mathrm{a}}$ and Anatomical Pathology, ${ }^{\mathrm{b}}$ Sir Charles Gairdner Hospital, ${ }^{\mathrm{c}}$ and the School of Population Health, University of Western Australia, Perth, Australia.

Read at the Eighty-sixth Annual Meeting of The American Association for Thoracic Surgery, Philadelphia, Pa, April 29-May 3, 2006.

Received for publication May 1, 2006; revisions received Dec 4, 2006; accepted for publication Dec 12, 2006.

Address for reprints: J. M. Alvarez, MBBS, FRACS, Clinical A/Professor Surgery, University of Western Australia, Department of Cardiothoracic Surgery, Sir Charles Gairdner Hospital, Verdun St, Nedlands, 6010, Perth, W. Australia, Australia (E-mail: John. Alvarez@health.wa.gov.au).

J Thorac Cardiovasc Surg 2007;133:1439-47 0022-5223/\$32.00

Copyright () 2007 by The American Association for Thoracic Surgery

doi:10.1016/j.jtcvs.2006.12.061
Objectives: Idiopathic postpneumonectomy pulmonary edema is a leading cause of mortality after pneumonectomy. Postoperative hyperinflation of the remaining lung is an etiologic factor. We have demonstrated avoidance of postpneumonectomy pulmonary edema solely by changing management of the pneumonectomy space to a balanced drainage system. In sheep, we tested the following hypothesis: (1) Postoperative induced hyperinflation of the remaining lung can cause postpneumonectomy pulmonary edema. (2) A balanced drainage system can prevent its development.

Methods: We performed 37 right-sided pneumonectomies in adult sheep. In experiment 1 , after surgery, 10 sheep had continuous suction $(5 \mathrm{kPa})$ applied through an intercostal catheter placed in the empty hemithorax to induce mediastinal shift and hyperinflation of the left lung without adverse hemodynamic sequelae. In experiment 2, 27 sheep were randomly allocated into 3 equal groups regarding management of the residual empty right hemithorax: balanced drainage, no intercostal drainage, and clamp-release intercostal underwater drainage. A fourth group of 9 sheep served as a sham controls placebo with the same anesthetic and a right thoracotomy.

Results: All sheep tolerated surgery without adverse event. In experiment 1, there was significant mediastinal shift at necropsy in all sheep and 60\% $(\mathrm{n}=6)$ had postpneumonectomy pulmonary edema develop in the left lung $(P=.023$ vs sham). In experiment 2 , incidences of postpneumonectomy pulmonary edema were as follows: 0 in balanced group ( $P=.057$ vs other groups), 3 (30\%) in no-drainage group, and $3(30 \%)$ in clamp-release group. Only the 12 sheep with postpneumonectomy pulmonary edema had respiratory distress; the rest had uneventful recoveries.

Conclusion: In a sheep model of postpneumonectomy pulmonary edema, hyperinflation from mediastinal shift is an etiologic factor. A balanced drainage system averts postpneumonectomy pulmonary edema. This is the first time such a causal relationship has been demonstrated, supporting our continued use of balanced drainage after pneumonectomy.

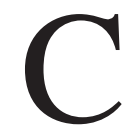
urrently, pneumonectomy remains associated with high perioperative mortality (approximately 10\%), regardless of where or by whom it is performed. ${ }^{1}$ Many contributing factors to this peripneumonectomy mortality are immutable (age of patient, site of pathology [right vs left]). A leading cause of mortality, however, is the development of idiopathic postpneumonectomy pulmonary edema (PPE). ${ }^{2,3}$ Potential etiologic variables for PPE, particularly if alterable (such as the perioperative management of the empty hemithorax), demand analysis. 


\section{Abbreviations and Acronyms}

ICT $=$ intercostal tube

PPE = postpneumonectomy pulmonary edema

UWS $=$ underwater seal

One such hypothetic variable is inadvertent postoperative hyperinflation trauma to the remaining lung. The mechanism of this hyperinflation may be from induced mediastinal shift toward the empty hemithorax as a result of reduced intrahemithoracic pressure from the egress of air (such as after coughing or straining) from the empty hemithorax. Two clinical reports have implicated postoperative hyperinflation as an etiologic factor for PPE., ${ }^{4,5}$ Since 1996, we have abolished the incidence of PPE by adopting a balanced drainage system for the management of the residual empty hemithorax. ${ }^{5}$ This system, described in $1955^{6}$ and designed to prevent hyperinflation of the remaining lung (as a result of mediastinal shift), is infrequently used.

In a balanced drainage system (Figure 1), the draining intercostal tube (ICT) empties into a collecting chamber. This chamber is connected by a Y-piece to a parallel system of predetermined positive and negative pressure regulation. The positive pressure regulator is a simple underwater seal (UWS) system. The distance the chest tube runs underwater determines the required level of positive pressure for air to leave the chest. Conversely, the negative pressure regulator determines (by the distance the air tube is underwater) at what negative pressure within the chest air enters from the atmosphere. This system allows automatic correction of intrathoracic pressure in the empty hemithorax, thus preventing mediastinal shift.

We therefore aimed to test, in sheep, the following hypotheses: (1) After a right pneumonectomy, the continuous application of $5 \mathrm{kPa}$ suction to this hemithorax through an ICT would produce PPE by inducing mediastinal shift and thus secondary hyperinflation of the left lung. (2) After a right pneumonectomy, a balanced drainage system would avoid PPE relative to traditional methods of managing the pneumonectomy space.

\section{Materials and Methods}

We received approval from the animal care and ethics committee of the University of Western Australia for these experiments; all animals were treated to animal care and ethics committee's satisfaction.

\section{Experiment 1: After Right Pneumonectomy, Can} Induced Hyperinflation of the Left Lung Cause PPE?

Surgery. After a 2-week quarantine, 10 adult merino sheep underwent a right-sided pneumonectomy. The sheep were weighed before fasting and on the morning of the operation. Before anesthetic induction, the sheep were sedated with ketamine $(100 \mathrm{mg} /$ $\mathrm{mL}$; Troy Laboratories Pty Ltd, Smithfield, New South Wales, Australia) at a dose of $11 \mathrm{mg} / \mathrm{kg}$ administered intramuscularly. Anesthetic induction was achieved with $100-\mathrm{mg} / \mathrm{mL}$ xylazine hydrochloride (Xylazil-100; Troy Laboratories) at a dose of 0.22 $\mathrm{mg} / \mathrm{kg}$ administered intramuscularly. The sheep were then intubated with a single-lumen tube (Portex; Mallinckrodt Laboratories Ltd, Athlone, Ireland). Anesthesia was maintained with halothane $1.5 \%$ to $2 \%$ (Rhodra-Halothane; Mercal Australia, Parramatta, New South Wales, Australia).

An Ohmeda 7000 ventilator (BOC Healthcare Group, Madison Wis) delivered tidal volumes of $6 \mathrm{~mL} / \mathrm{kg}$, with respiratory rates of

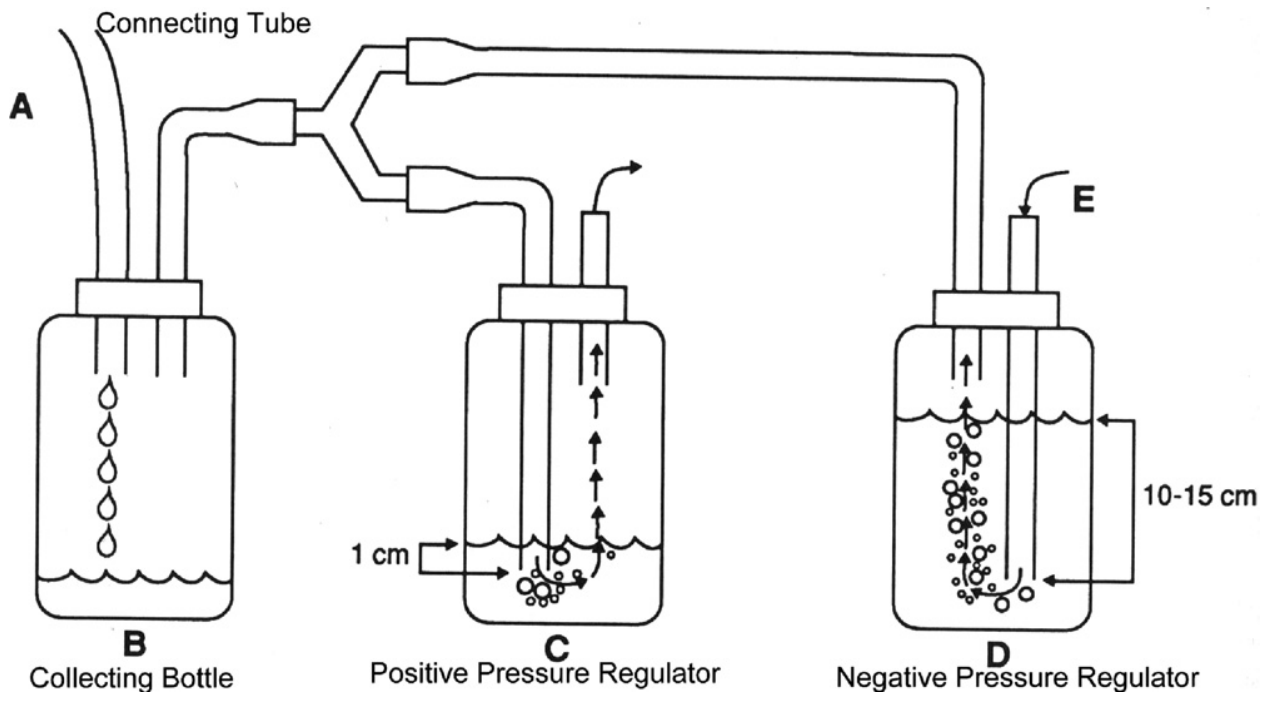

Figure 1. Balanced drainage system. $A$, Draining intercostal tube; $B$, collecting chamber; $C$, positive pressure regulator; $D$, negative pressure regulator; $E$, air tube. 
14 to 16 breaths/min, an inspiratory/expiratory ratio of $1: 2$, and oxygen delivery at an inspired oxygen fraction of $100 \%$ [Medical EPGrade Oxygen 131262; BOC Gases, Murray Hill, NJ]. No muscle paralyzing drugs were used. Pulse oximetry obtained with a Nellcor N-180 pulse oximeter (Nellcor Puritan Bennett Inc, Pleasanton, Calif) and electrocardiographic monitoring were available for all sheep. Intraoperatively, $0.9 \%$ normal saline solution (Baxter Healthcare Australia and New Zealand, Old Toongabbie, New South Wales, Australia) intravenously was titrated to match estimated overnight fast and operative loss (blood and gastric fluid). Antibiotic cover was administered to all sheep before skin incision with a single $1-\mathrm{mL} / 10 \mathrm{~kg}$ dose of oxytetracycline dihydrate $(200 \mathrm{mg} / \mathrm{mL}$; Norbrook Australia, Tullamarine, Victoria, Australia) administered intramuscularly.

Through a right 5 th interspace thoracotomy, the chest was entered. The superior and lower bronchi were dissected, divided, and stapled with the Proximate TX 30G linear stapler (Ethicon Endo-Surgery, Inc, Cincinnati, Ohio), thus allowing collapse of the lung to occur. The hilar vessels were then dissected, ligated, or stapled with the Proximate TX 30V linear stapler and divided, with the pulmonary artery and its branches preceding the individual pulmonary veins. No mediastinal dissection was performed in any animal.

The chest was closed with a series of four separate 1-0 nylon (Ethilon; Johnson \& Johnson, Markham, Ontario, Canada) pericostal sutures, the two muscle layers were closed with a continuous suture of 2-0 polypropylene (Prolene; Ethicon, Inc, Somerville, $\mathrm{NJ}$ ), and the skin was closed with a continuous 5-0 nylon (Ethilon). No wound dressings were applied.

A single ICT (Portex Standard; Sims Portex Ltd, Hythe, UK) was inserted through the 6th interspace, positioned to lie ventrally, and connected to $5-\mathrm{kPa}$ continuous wall suction until the animal was killed. The sheep were monitored for 1 hour before leaving the operating theater to ensure that no adverse hemodynamic or respiratory sequelae occurred.

The sheep were housed in individual monitoring pens with an adjacent partner. Twice daily clinical parameters were measured. Every 6 to 8 hours, $1 \mathrm{~mL}$ buprenorphine hydrochloride (Temgesic $324 \mu \mathrm{g}$; Reckitt Benckiser Healthcare UK Ltd, Hull, UK) was administered according to agreed protocols.

The sheep were humanely killed according to animal care and ethics committee protocols 5 days after surgery or on the development of clinically severe respiratory distress. For humane death, the sheep were sedated with ketamine $(100-\mathrm{mg} / \mathrm{mL}$; Troy Laboratories) at a dose of $11 \mathrm{mg} / \mathrm{kg}$ administered intramuscularly, followed by intracardiac $20 \mathrm{~mL}$ pentabarbitone sodium $(325-\mathrm{mg} / \mathrm{mL}$ Lethabarb; Virbac [Australia] Pty Ltd, Peakhurst, New South Wales). An immediate standardized necropsy was performed. The right side of the chest was inspected, followed by the left, including pneumonectomy and cardiectomy.

Histopathologic analysis. The two pathologists (A.S. and G.S.) were blinded to the clinical course of all sheep. Pulmonary edema was defined by the presence of pale amorphous, acellular, eosinophilic material within the alveolar spaces. ${ }^{7}$ PPE was graded on a scale comprising 0 (no edema fluid), 1 (occasional patchy foci), 2 (widespread), and 3 (widespread and marked). PPE was considered present with grade 2 to 3 changes. This method was developed by us a priori.
The right lung was drained of blood, weighed, and delivered for histopathologic analysis. The lung was gravity filled through the main bronchus to the main bronchial margin with neutral buffered formalin (CONFIX 10\% formaldehyde with sodium phosphonate buffer salts; Amler Scientific P/L, Belmont, West Australia, Australia) diluted 1:4 with water before a standardized protocol for division (6-8 slices from base to apex) and hematoxylin and eosin staining.

At necropsy the left lung was removed, drained of blood, weighed, and prepared the same as the right lung. All lungs were fixed in CONFIX within 1 hour from explantation.

The statistical method used to measure agreement between the pathologists for the presence of PPE was the $\kappa$ variable, defined as the agreement beyond chance divided by the amount of possible agreement beyond chance. ${ }^{8}$

\section{Experiment 2: After Right Pneumonectomy, Can the Surgical Management of the Empty Hemithorax Influence the Occurrence of PPE?}

Thirty-six sheep were prospectively randomly assigned (by a closed-envelope system) into four groups according to contemporary options for postoperative management of the empty right hemithorax. Anesthetic, perioperative fluid regimen, and surgery were standardized as in experiment 1 . Twenty-seven sheep underwent a right pneumonectomy in equal groups of 9: group A had an ICT placed in the right side of the chest at surgery and attached to a balanced drainage system $\left(-13 \pm 1 \mathrm{~cm} \mathrm{H} \mathrm{H}_{2} \mathrm{O}\right.$, Pleur-Evac; Deknatel, Inc, Fall River, Mass), group B had no ICT placed in the right side of the chest, and group $\mathrm{C}$ had an ICT placed in the right side of the chest at surgery and attached to UWS drainage. This ICT was clamped from the time of surgery, only being released for 2 minutes at a time 6 times per day. A sham operation group underwent 60 minutes of anesthesia, right thoracotomy, and wound closure without ICT placement (group D). Necropsy and pathologic assessment were as in experiment 1.

\section{Results}

\section{Experiment 1}

Table 1 delineates the physical characteristics of this group. There were no perioperative complications. Intraoperative blood loss ranged from 100 to $200 \mathrm{~mL}$ (mean $150 \pm 20 \mathrm{~mL}$ ). Perioperative mean intravenous fluid load was $600 \pm 100 \mathrm{~mL}$ (300-700 mL) during 5 hours. The sheep tolerated pneumonectomy and the application of 5-kPa suction uneventfully.

No demonstrable hemodynamic compromise was evident immediately after surgery. In particular, in all sheep immediately postoperatively, no differences were evident in each animal's demeanor, feeding habits, passage of stool and urine, respiratory rate, heart rate, temperature, and strength of carotid pulsations (ie, normal, reduced, or absent) relative to its own characteristics before surgery or those of its pen companion.

From 5 to 72 hours after surgery, however, 6 of the 10 sheep had clinical features of respiratory distress develop: respiratory rate greater than 50 breaths/min; panting; and inability to normally feed, drink, or move like the paired 
TABLE 1. Sheep characteristics: Experiment 1

\begin{tabular}{rlcccc}
\hline & & \multicolumn{3}{c}{ Weight } & \\
\cline { 3 - 5 } Sheep & Sex & Total $(\mathbf{k g})$ & Right lung $(\mathbf{m g})$ & Left lung $(\mathbf{m g})$ & Postpneumonectomy pulmonary edema \\
\hline 1 & Female & 34 & 228 & 186 & Yes \\
2 & Male & 38 & 296 & 266 & Yes \\
3 & Male & 46 & 324 & 349 & No \\
4 & Male & 39 & 344 & 310 & Yes \\
5 & Female & 30 & 276 & 248 & No \\
6 & Female & 30 & 287 & 149 & No \\
7 & Male & 34 & 229 & 310 & Yes \\
8 & Male & 32 & 213 & 162 & Yes \\
9 & Female & 35 & 291 & 231 & No \\
10 & Male & 35 & 246 & 240 & Yes \\
\hline
\end{tabular}

companion. Two sheep had to be killed at 5 and 48 hours after surgery because of progressive and severe respiratory distress. The remaining 8 sheep were killed as planned on day 5. The left lungs of all affected sheep appeared abnormal, with features of congestion and pink fluid present in the major bronchi. The degree of pulmonary edema ranged from severe PPE (grade 3, 5 sheep, see Limitations section) to moderate (grade 2, 1 sheep). The pattern of distribution was generalized in 5 and segmental in 1.

At necropsy, in all sheep there were no abnormalities in the operated hemithorax; in particular, there was no evidence of hemorrhage or infection, and the bronchial and vascular stumps were intact. The pneumonectomy space was significantly reduced, at times nearly obliterated, in all cases from predominantly mediastinal but also diaphragmatic displacement. Subcutaneous emphysema to a variable degree was evident in all sheep without a balanced drainage system, particularly those in which PPE developed. It was, however, impossible to quantify accurately the magnitude and extent of this emphysema. The heart and great vessels were excised and examined macroscopically; all were normal, in particular, there was no evidence of pulmonary embolism or myocardial pathology (infarction, edema, hyperemia). In all animals, visual inspection of the major epicardial coronary arteries, including transverse sections of the heart, confirmed the lack of evidence of epicardial coronary artery disease. There was no clinical evidence of right heart failure (peripheral edema, conjunctival edema, ascites).

\section{Experiment 2}

All 36 sheep tolerated the surgery without adverse event. The physical characteristics (sex, weight) of these 36 sheep were not significantly different among the four groups, nor from those in experiment 1 . All 36 right lungs exhibited normal histologic characteristics. At necropsy, no abnormalities in the operated hemithorax, the heart, and the pulmonary vasculature were detected. No sheep in groups A or D exhibited respiratory distress. The left lungs of all sheep in groups A and D were normal.

In groups B and C, 3 sheep in each group had respiratory distress develop; none died or had to be killed before schedule. Each of these 3 sheep in each group had abnormal left lung histopathologic appearance. One sheep in each group had severe PPE (grade 3) (Figure 2), and 2 sheep per group had moderate PPE (grade 2) (Figure 3).

There were no significant differences regarding physical characteristics, operating time, and intravenous fluid load for any group. There was a significant difference in the incidence of PPE in sheep in experiment $1(P=.023)$. We did not reach statistical significance when comparing the incidence of PPE according to the method of management of the empty chest $(P=.057$; Table 2$)$.

\section{Limitations}

We faced constraints in performing these experiments because of logistics, costs, and animal welfare considerations.

Based on an expected incidence of PPE of 5\% after right pneumonectomy, to statistically detect a difference for $\alpha=$

TABLE 2. Group comparisons

\begin{tabular}{lccc}
\hline & $\begin{array}{c}\text { Pulmonary } \\
\text { edema }\end{array}$ & $\begin{array}{c}\text { No pulmonary } \\
\text { edema }\end{array}$ & Total \\
\hline Experiment 1 & 5 & 5 & 10 \\
Experiment 2 & & 9 & 9 \\
Balanced drainage (group A) & 0 & 6 & 9 \\
No intercostal tube (group B) & 3 & 6 & 9 \\
Intercostal tube with & 3 & 9 & 9 \\
$\quad$ underwater seal (group C) & & & \\
Sham operation (group D) & 0 & 9
\end{tabular}

All values represent numbers of sheep. For comparison of all groups in experiments 1 and 2, Pearson $\chi^{2}{ }_{4}=10.28$, Fisher exact test $P=.023$. Note only 5 sheep with postpneumonectomy pulmonary edema confirmed by histopathologic examination are included for statistics in experiment 1. For comparison of groups in experiment 2, Pearson $\chi_{3}^{2}=7.2$, Fisher exact test $P=.057$ 
$.05, \beta=.10$, and $80 \%$ power, we calculated the number of sheep required for a given incidence of PPE. For a 50\% rate of PPE, 11 sheep were required for experiment 1 . For experiment 2, for an incidence of PPE of 30\%, 21 sheep per group were required. We were thus underpowered in experiment 2. Pragmatically, we stopped experiment 2 at 9 animals per group because of costs; we considered clinical relevance to be evident at this point (Table 2).

The diagnosis of PPE was based on histologic examination by liquid formalin bronchial inflation, the only method available to us. This technique likely results in underestimation of the true incidence of PPE, because it may reduce the amount of edematous material visible in the alveolus. ${ }^{9}$ Furthermore, interstitial alveolar wall edema, an earlier and more accurate marker, could not be measured. ${ }^{9}$ In 1 sheep (experiment 1, distress 5 hours after surgery), the diagnosis could not be made by histopathology (sheep killed after laboratory hours). The clinical findings of gross pink frothy fluid in a heavily congested lung, however, strongly suggest PPE.

We were unable to measure the magnitude of volumepressure changes within the chest and correlate these with histopathologic findings. Mediastinal shift was clearly evident at necropsy, but left lung hyperinflation could only be deduced from necropsy findings. There was a clear impression, however, that left lung hyperinflation was present, particularly relative to our sham operation group. A pilot study revealed the inadequacy of performing serial chest radiography in sheep. Absence of myocardial damage and infection was deduced on clinical grounds only. Respiratory distress in sheep, unless severe, is difficult to quantify perioperatively; only the presence of continuous panting, reduced feeding, and prolonged lying relative to sheep not operated on appeared to correlate with PPE.

In sheep, the chest and mediastinum are highly compliant, so uniform and substantial distension of the lung was evident. The magnitude of this parenchymal stretch is unlikely to be replicated in the human patient undergoing surgery for lung cancer. The magnitude of mediastinal shift tolerated by sheep without evident hemodynamic compromise may have a significant effect on elevating central venous and lymphatic pressures. These factors may themselves cause pulmonary edema, with or without associated lung hyperinflation. Our experiments cannot exclude such confounding variables in the etiology of PPE.

We have been unable to elucidate the minimum time required to maintain mediastinal equilibrium postoperatively to prevent PPE.

\section{Discussion}

The continuous application of 5-kPa suction to the empty hemithorax after right pneumonectomy in sheep induces significant mediastinal shift, with near obliteration of the empty hemithorax but without hemodynamic compromise. We deduced that hyperinflation of the remaining lung is associated with the development of significant and severe acute pulmonary edema within 5 days after surgery in $60 \%$ of sheep.

The postoperative management of the empty hemithorax after right pneumonectomy appears to have a significant bearing on causing PPE. According to histopathologic examination, 30\% of sheep managed after a right pneumonectomy by a protocol of either clamp-release ICT or no ICT had PPE develop. No sheep with a balanced drainage system (group A) or sham operation (group D), however, had PPE develop (Figure 4).

It is generally agreed that PPE appears to be multifactorial in etiology; exhaustive reviews have detailed potential variables. ${ }^{10,11}$ These variables may be associated with anesthesia, surgery, and postoperative management. The performance of a right-sided pneumonectomy has consistently been demonstrated to be a major risk factor, with a 3- to 5-fold increased risk of PPE relative to a left-sided operation. ${ }^{12}$ We therefore performed right-sided pneumonectomies, because the magnitude of induced hyperinflation of the remaining lung would be larger than with the converse operation. If hyperinflation proved to be an etiologic variable, this approach should improve the chances of PPE occurring. Furthermore, by excluding or minimizing other known etiologic variables, our hypotheses could be tested with a reduced likelihood of being confounded.

No single factor has caused greater controversy between surgeon and anesthetist than the recommendation by Zeldin and colleagues, ${ }^{13}$ "The most important thing that we can do in terms of recognizing this problem is to watch our anesthetists as they start loading the patient up with fluid." Therefore in particular, all sheep were not subjected to intravascular volume expansion; they were kept dry. Intravenous volume in the perioperative period (5 hours) was matched to estimated fasting weight, gastric, and intraoperative blood losses; mean crystalloid volume replacement was $600 \mathrm{~mL}$. It is important to reiterate that we, like others, have previously demonstrated that PPE will occur despite keeping patients dry after pneumonectomy. ${ }^{4,5}$

Similarly, other surgical variables associated with PPE were excluded. ${ }^{11}$ Namely, operative times were brief, no mediastinal or carinal resections were performed, blood loss was small, no homologous transfusions were given, and no ribs were fractured (thus creating no fat emboli). At necropsy, no relevant pathologic conditions were present (empyema, bronchopleural fistulas, pulmonary emboli, cardiac failure or infarction). In addition, no pharmacologic agent implicated in the etiology of PPE was used (eg, amiodarone).

Importantly, we excluded anesthetic variables implicated in the cause of PPE. ${ }^{10}$ Namely, we used physiologic tidal volumes $(6 \mathrm{~mL} / \mathrm{kg})$ through a single-lumen endotracheal 


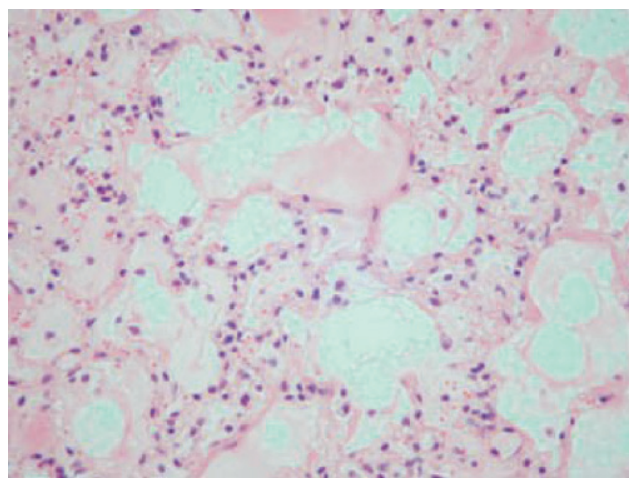

Figure 2. Postpneumonectomy pulmonary edema grade 3 (original magnification $\times 240$ ). Widespread and severe pulmonary edema (pink proteinaceous fluid within alveolar air sacs). Generalized congestion of alveolar walls and capillaries.

tube. Currently, many anesthetists use tidal volumes on single-lung ventilation as high as $10 \mathrm{~mL} / \mathrm{kg}$. This factor may lead to high intraoperative inspiratory airway pressures $\left(>30 \mathrm{~cm} \mathrm{H}_{2} \mathrm{O}\right.$ ), which have been implicated in the cause of PPE. By using physiologic tidal volumes, this variable has been minimized if not abolished. We were unable to repeatedly measure peak inspiratory airway pressures; on the occasions we did, however, peak inspiratory pressures were low $\left(<10 \mathrm{~cm} \mathrm{H}_{2} \mathrm{O}\right) .{ }^{12}$

Impressively, more than half of the young, healthy animals with normal pulmonary and cardiac function had PPE develop in response to induced mediastinal shift and hyperinflation of the left lung. Although we were unable to measure the magnitude of left lung hyperinflation, at necropsy it was evident and substantial.

There exists supporting evidence from three sources (in experiments related to congenital diaphragmatic hernia) that

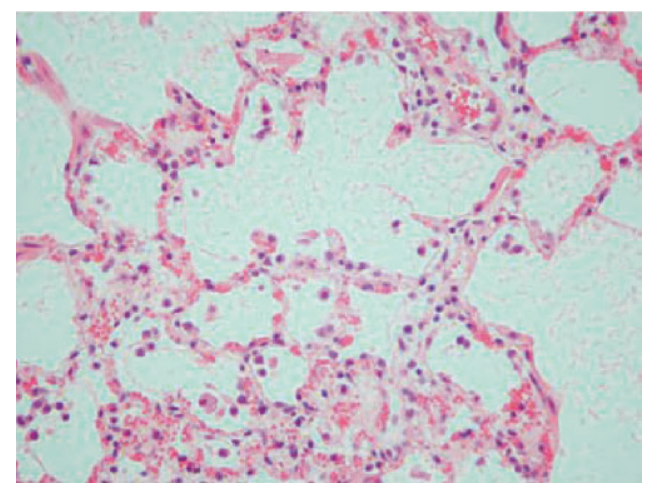

Figure 3. Postpneumonectomy pulmonary edema grade 2 (original magnification $\times 240$ ). Widespread moderate pulmonary edema within alveolar air sacs. Generalized congestion of alveolar walls and capillaries.

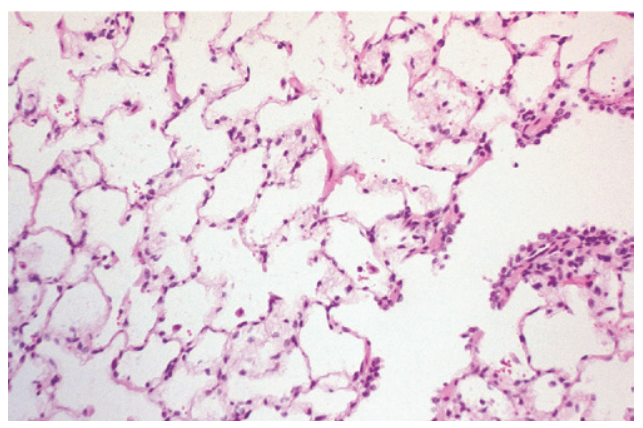

Figure 4. Postpneumonectomy pulmonary edema grade $\mathbf{0}$, normal lung (original magnification $\times 130$ ). No edema fluid present in alveolar air sacs. Normal alveolar walls, no capillary congestion evident.

such hyperinflation is associated with PPE. Ramenofsky ${ }^{14}$ divided newborn puppies subjected to left pneumonectomy into two groups according to the management of the ICT placed in the left side of the chest. Group 1 had the ICT drained to UWS at $-2 \mathrm{~cm}_{2} \mathrm{O}$, and group 2 had the ICT attached to a pressure manometer and air-filled syringe, preventing mediastinal shift by air injection. At 24 hours after the operation, all group 1 animals had died of histologically confirmed adult respiratory distress syndrome, whereas there were no such deaths in group 2. Raffensperger and coworkers ${ }^{15}$ demonstrated that the removal of air from the empty hemithorax after left pneumonectomies on puppies produced immediate hypoxemia, hypercapnia, and increased alveolar-arterial carbon dioxide gradients. The removal of such air caused mediastinal shift and hyperinflation of the remaining lung, with histologically confirmed interstitial emphysema and disrupted alveoli. Raffensperger and coworkers ${ }^{15}$ therefore advocated midline mediastinal stabilization after repair of congenital diaphragmatic hernia. De Luca and associates ${ }^{16}$ noted that interstitial emphysema developed in all fetal lambs with surgically created congenital diaphragmatic hernia followed by delayed repair, with ICT drainage promoting barotrauma from hyperinflation of the remaining lung.

Furthermore, two clinical reports support our hypothesis. Deslauriers and colleagues, ${ }^{4}$ in a series of 291 pneumonectomies (1988-1993), identified three variables implicated in PPE, and the type of postoperative pleural drainage system used was a significant risk factor $(P=.009)$. No multivariate analysis was reported regarding the other two variables, extent of resection and length of surgery. Nevertheless, no patient $(0 / 77)$ with a balanced drainage system had PPE develop, versus $2.6 \%$ (2/78) with no ICT drainage and 9.1\% (11/121) with ICT to UWS drainage.

We changed from a system of ICT to UWS drainage clamped with intermittent release (1993-96) to a balanced 
drainage system (1996-2000). ${ }^{5}$ Our incidence of PPE changed from $14.3 \%$ (4/28 pneumonectomies, 1993-1996) to $0 \%(0 / 29$ pneumonectomies, $1996-2000, P=.001) .{ }^{5} \mathrm{We}$ have had no further cases of PPE since this change. Importantly, no case of postpneumonectomy space infection has occurred in our 10-year experience, nor were any reported by either Laforet and Boyd ${ }^{6}$ or Deslauriers and colleagues. ${ }^{4}$

Two potential etiologic variables could not be excluded, however: intraoperative hyperoxia and postoperative hypoxia. Logistically, we were unable to minimize the effect of intraoperative hyperoxia. ${ }^{17}$ We could only deliver a $100 \%$ inspired oxygen fraction. The same inspired oxygen fraction was used in all 46 sheep, however, and operation times were short. Furthermore, Reed and colleagues ${ }^{18}$ demonstrated that significant right ventricular dysfunction does occur after pneumonectomy and that conventional indices of right ventricular performance do not reflect this cardiac impairment. Slinger ${ }^{12}$ therefore cautions against premature withdrawal of oxygen therapy after surgery because it may exacerbate right ventricular dysfunction. It was impossible to provide sheep with oxygen therapy. Unlike the patients of Reed and colleagues, ${ }^{18}$ however, these were young subjects with normal cardiorespiratory function and without mediastinal node dissection being performed.

Thus, within the constraints of experiment 1 we demonstrated that mediastinal shift with hyperinflation of the remaining lung after pneumonectomy is associated with a high rate of PPE. We also demonstrated that the surgical management of the pneumonectomy space has a significant bearing on the histopathologic incidence of PPE. In these experiments, management of the empty hemithorax by either no chest ICT or ICT clamp with intermittent release did not abolish the occurrence of PPE. In contrast, a balanced drainage system did so.

In experiment 2, no sheep had to be killed prematurely because of respiratory distress. In experiment 1 , however, 2 sheep were killed early because of severe respiratory distress, and 3 others would have been had it not already been day 5. Sheep with respiratory distress in experiment 1 appeared to have more severe conditions than those in experiment 2. It is impossible to know whether grade $2 \mathrm{PPE}$ would have progressed to clinical PPE. Yet this may be important; in a radiologic study, van der Werff and coauthors ${ }^{19}$ noted that $33 \%$ of human patients had a radiologically apparent but subclinical syndrome of pulmonary edema after pneumonectomy, but only a minority (5.6\%) had actual progression to clinical PPE.

This factor may explain the difference in our reported incidence of PPE according to histopathologic examination in experiment $2(30 \%)$ and the clinical incidence in human beings of $5 \%$ to $9 \%$. Also sheep, as we hypothesize on the basis of our findings of subcutaneous emphysema at necropsy, may be prone to inducing the egress of air from an empty hemithorax and subsequent left lung hyperinflation as a result of their nearly continuous postoperative bleating, which inexorably requires compression forces applied to the entire chest wall.

We were not able to demonstrate either the actual pathologic cause for PPE or the time of onset. We surmise that there is damage to the alveolar endothelial lining. The pattern of damage appears to be multifocal. Waller and associate ${ }^{20}$ demonstrated in human beings by scintigraphy with radioactively labeled albumin that the remaining lung after pneumonectomy has increased permeability. Because a normal lung is maximally compliant at its functional residual capacity, it is plausible that any further inflation under the acute stress of receiving the entire cardiac output after pneumonectomy could trigger disruption of lung parenchyma. In humans requiring surgery, additional factors of postoperative right ventricular dysfunction, abnormal lung parenchyma from smoking, and reduced lymph flow reserve are common. It is thus unsurprising that the imposition of a further physiologic insult (hyperinflation) could trigger PPE.

Interestingly, the normal residual animal lung after pneumonectomy exhibits enormous reserve in resisting the occurrence of pulmonary edema when subjected to severe cardiovascular insults. Zeldin and colleagues ${ }^{13}$ subjected dogs to right pneumonectomy and massive intravenous crystalloid challenges (acute $100 \mathrm{~mL} / \mathrm{kg}$ before surgery and $100 \mathrm{~mL} / \mathrm{kg}$ positive fluid balance over 48 hours, ) yet more than half of the animals $(62.5 \%)$ did not have edema develop. Zarins and associates ${ }^{21}$ induced a 7 -fold increase in lymphatic drainage in response to a 76\% lowering of plasma oncotic pressure, yet no animal had edema develop. Little and coworkers ${ }^{22}$ noted no difference in the increase in extravascular lung water between dogs undergoing pneumonectomy with or without mediastinal nodal dissection acutely challenged with a crystalloid load to a left atrial pressure of $23 \mathrm{~mm} \mathrm{Hg}$. This reserve is not evident under similar circumstances, however, when the insult is caused by hyperinflation. Thus in experiment 1 , a modest change in intrathoracic pressure $(-5 \mathrm{kPa})$ produced a high rate of PPE (60\%). Similarly, Ramenofski ${ }^{4}$ with an ICT set to $-2 \mathrm{~cm}$ $\mathrm{H}_{2} \mathrm{O}$ observed a $100 \%$ rate of fatal adult respiratory distress syndrome by 24 hours. Interestingly in that study, recorded intrapleural pressure differences exceeded $-10 \mathrm{~cm} \mathrm{H}_{2} \mathrm{O}$ by 4 hours after surgery despite the ICT to UWS drainage.

Our findings therefore appear relevant. In human beings, PPE is not uncommon and carries a very high mortality. Although many etiologic factors are immutable, how we manage the pneumonectomy space is not. A crucial postoperative management factor is the prevention of mediastinal shift. This requires both recognition of mediastinal shift and treatment (injection of air into the hemithorax). A balanced drainage system allows continuous correction of 
pressure changes in the empty hemithorax. Furthermore, in light of the inherent weaknesses of the alternative options (ICT clamp-release, ICT to UWS, no ICT), a balanced drainage system appears most effective. This has been our clinical experience.

Previously, we mentioned that either a randomized, controlled clinical trial or an animal model would validate or repudiate our hypothesis and experience. ${ }^{5}$ Pragmatism suggests that such a clinical trial, although desirable, is unlikely. On the basis of our clinical experience and these experiments, we continue to advocate managing the empty hemithorax after pneumonectomy with a balanced drainage system.

We would like to thank Dr Cyrus Ediban, Specialist in Intensive Care, Royal Perth Hospital and Mr. Anthony Wilson, Cardiothoracic Surgeon, St. Vincent's Hospital, Melbourne, for their insights into this challenging problem.

\section{References}

1. Fuentes PA. Pneumonectomy: historical perspective and prospective insight. Eur J Cardiothorac Surg. 2003;23:439-45.

2. Slinger P. Post-pneumonectomy pulmonary edema: is anesthesia to blame? Curr Opin Anaesthesiol. 1999;12:49-54.

3. Alvarez JM, Bairstow BM, Tang C, Newman MA. Post-lung resection pulmonary edema: a case for aggressive management. $J$ Cardiothorac Vasc Anesth. 1998;12:199-205.

4. Deslauriers J, Aucoin A, Gregoire J. Postpneumonectomy pulmonary edema. Chest Surg Clin North Am. 1998;8:611-31.

5. Alvarez JM, Panda RK, Newman MA. Postpneumonectomy pulmonary edema. J Cardiothorac Vasc Anesth. 2003;17:388-95.

6. Laforet EG, Boyd TF. Balanced drainage of the postpneumonectomy space. Surg Gynaecol Obstet. 1964;18:1051-64.

7. Slavin G, Kreel L, Herbert A, Sandin B. Pulmonary oedema at necropsy: a combined pathological and radiological method of study. J Clin Pathol. 1975;28:357-66.

8. Dawson B, Trapp RG. Research questions about one group. In: Dawson B, Trapp RG. Basic and clinical biostatistics. 3rd ed. Singapore: McGraw-Hill International Editions; 2001. p. 115-7.

9. Hammond TG, Mobbs M. Lung oedema-microscopic detection. J Appl Toxicol. 1984;4:219-21.

10. Slinger PD. Perioperative fluid management for thoracic surgery: the puzzle of postpneumonectomy pulmonary edema. J Cardiothorac Vasc Anesth. 1995;9:442-51.

11. Alvarez JM. Chapter 9: post pneumonectomy pulmonary edema. In: Progress in thoracic anesthesia. Society of cardiovascular anesthesiologists monograph. Baltimore: Lippincott, Williams and Wilkins; 2004. p. $187-220$.

12. Slinger PD. Postpneumonectomy pulmonary edema: good news, bad news. Anesthesiology. 2006;105:2-5.

13. Zeldin RA, Normandin D, Landtwing D, Peters RM. Postpneumonectomy pulmonary edema. J Thorac Cardiovasc Surg. 1984;87:359-65.

14. Ramenofsky ML. The effects of intrapleural pressure on respiratory insufficiency. J Pediatr Surg. 1979;14:750-6.

15. Raffensperger JG, Luck SR, Inwood RJ. The effect of overdistension of the lung on pulmonary function in beagle puppies. J Pediatr Surg. 1979;14:757-60.

16. de Luca U, Cloutier JM, Laberge L, Fournier L, Prendt H, Major D, et al. Pulmonary barotrauma in congenital diaphragmatic hernia: experimental study in lambs. J Pediatr Surg. 1987;22:311-6.

17. Shimizu T, Abe K, Kinouchi K, Yoshiya I. Arterial oxygenation during one lung ventilation. Can J Anaesth. 1997;44:1162-6.

18. Reed CE, Spinale FG, Crawford FA Jr. Effect of pulmonary resection on right ventricular function. Ann Thorac Surg. 1992;53:578-82.
19. van der Werff YD, van der Houwen HK, Heijmans PJ, Duurkens VA, Leusink HA, van Heesewijk HP, et al. Postpneumonectomy pulmonary edema. A retrospective analysis of incidence and possible risk factors. Chest. 1997;111:1278-84.

20. Waller DA, Keavey P, Woodfine L, Dark JH. Pulmonary endothelial permeability changes after major lung resection. Ann Thorac Surg. 1996;61:1435-40.

21. Zarins CK, Rice CL, Peters RM, Virgilio RW. Lymph and pulmonary response to isobaric reduction in plasma oncotic pressure in baboons. Circ Res. 1978;43:925-30.

22. Little AG, Langmuir VK, Singer AH, Skinner DB. Hemodynamic pulmonary edema in dog lungs after contralateral pneumonectomy and mediastinal lymphatic interruption. Lung. 1984;162:139-45.

\section{Discussion}

Dr Mark K. Ferguson (Chicago, Ill). Alvarez and coauthors are to be congratulated on a unique study that I think was carefully conducted on an important clinical problem. This complication doesn't happen commonly, but it is quite lethal when it does occur. As far as I can tell, this is the first experimental study to attempt to assess the role of pleural space drainage techniques in the etiology of this disorder. The results provide interesting, albeit preliminary, insights into the potential pathophysiology of this problem.

Dr Alvarez, in your initial experiment in which all animals underwent pleural drainage at $5 \mathrm{kPa}$ pressure, there was a $60 \%$ rate of clinical respiratory distress. I understand that you think that this model is a somewhat typical clinical situation, but the extreme mediastinal deviation that you describe is not typical of the clinical situation, and by my calculation the $5 \mathrm{kPa}$ is equivalent to more than $-50 \mathrm{~cm} \mathrm{H}_{2} \mathrm{O}$ suction. Now, could that amount of shift cause a substantial decrease in venous return, elevated venous pressure, and decreased lymphatic drainage from the lung? If so, this problem may be unrelated to hyperexpansion and so-called volotrauma.

In the second experiment, in which you used several different drainage techniques, there were several shortcomings that you yourself mentioned. It isn't intuitively obvious to me why lack of drainage leads to lung overexpansion. So I wonder whether there were clinical findings in these animals that could suggest a method for mediastinal shift and hyperinflation of the remaining lung, such as extensive subcutaneous emphysema. Similarly, when you unclamped the UWS periodically, did those animals exhibit a large amount of air flow through the chamber when that occurred?

In assessing the pulmonary edema, although the histologic method you described has been standardized through a number of decades, I'm wondering whether you considered correlating these findings with some other way of measuring lung water, such as wet weight/dry weight ratios.

Your findings are intriguing and provide a possible insight into the clinical observation that balanced drainage is a superior technique for managing the postpneumonectomy space. I encourage you to refine the technique to better explore this problem.

Dr Alvarez. Thank you for those kind comments. Yes, $5 \mathrm{kPa}$ is about $50 \mathrm{~cm} \mathrm{H}_{2} \mathrm{O}$. It's extreme. The whole point was to show that this actually could cause PPE. The whole idea of doing a right pneumonectomy and so forth was to give ourselves the best opportunity to see this. We faced tremendous criticism in doing these experiments, causing tremendous emotional and psychologic disturbance to the sheep's well-being, and the idea of doing an 
experiment in which an animal is supposed to get sick is difficult to get through the ethics committee.

Why $5 \mathrm{kPa}$ was really quite simple. At $5 \mathrm{kPa}$, the tubes collapsed. We couldn't go any higher. I think we're fortunate that the sheep have a tremendous mesentery between the inferior vena cava and the mediastinum. It's about 4 or $5 \mathrm{~cm}$ there. We really didn't know when we applied it whether we would have hemodynamic collapse and so forth, and we had to do a pilot study and so on. It doesn't happen. They cope with it remarkably well.

You comment on the lymphatics and the effects of right ventricular dysfunction, and you are quite right. These are potential confounding variables. But I think that what we showed was that in a young, healthy, sheep without exposure to cigarette smoke, you apply $5 \mathrm{kPa}$ of suction (and it may actually only take $3 \mathrm{kPa}$ or $2 \mathrm{kPa}$ ) to inflate that lung, and 6 of 10 sheep get sick and have PPE develop.

As to, why lack of drainage is associated with PPE, I suspect a possible reason is that if air comes out of a thoracic cavity, the most likely source is the chest, which can lead to mediastinal shift. You can have massive volume changes. And I suspect that may well be the case in our patients. They cough, they expectorate, all those things, and some air may move. I suspect that's the actual issue there. Air escaped. There is not a hermetic seal. Things change.

When we looked back, all of our cases of PPE had subcutaneous emphysema. As to whether I can correlate that with our sheep, it's quite hard to do, and I'm afraid I just couldn't do it. That's one of the limiting factors. How much air came out of the chest tube when we clamped and released the thing was impossible to determine. I couldn't do it all the time by myself. I had to rely on animal technicians and so on and so forth. I thus can't quantify that. There are certain limitations.

The right/left ratios weren't going to tell us much, because I was constrained by the ethics committee. The sheep had to be killed on day 5. Now, if I could examine these rations when there was a fatality, then we might be able to show something. So with these right/left ratios, there was no correlation. If the triggering event occurred, say, 24 hours before the animal was killed versus 2 or 3 days, you would have substantial differences with those right/left ratios.

Dr Frank Detterbeck (New Haven, Conn). I commend you on a great study and taking this issue to the laboratory to try to understand this. If we look in the literature and at our clinical experiences, there is also an incidence of unexplained pulmonary edema that occurs after less serious resections than pneumonectomy, such as after a lobectomy and even occasionally after a wedge resection. Furthermore, if you look at an extended pneumonectomy, such as a carinal sleeve pneumonectomy or something like that, the incidence is higher than for simple pneumonectomy. So the incidence seems to correlate with the magnitude of the operation. I'm having a hard time explaining pulmonary edema in the setting of a lobectomy by your findings regarding hyperinflation. Do you have thoughts regarding that?

Dr Alvarez. In our first article, 1 of the patients actually had undergone a lobectomy. It was a lower lobe, and a very big lower lobe at that. I think it doesn't happen as much with a lobectomy simply as a volume issue. But if you get the right circumstances, such as a large lower lobe and thus a potential space, it doesn't take much to push these patients over the edge. Their lymphatics may be interfered with, right ventricular dysfunction can occur, they are all smokers, and so on. We already know from Waller's experience that the lungs are leaky after pneumonectomy. If you add another insult in our patient population and somehow disrupt that alveolar epithelial membrane in whatever way, it's like a crack in a dam. So you get the right patient with a large lower lobe that you have taken out, and there's a potential space there. You have set yourself up for it. Now, a balanced drainage system is one way of alleviating this. This is a minute-by-minute, instantaneous measurement. 